\title{
Distrofia polimorfa posterior vista por medio de tomografía Scheimpflug y retroiluminación
}

\section{Posterior polymorphous corneal dystrophy as seen through Scheimpflug tomography and corneal retroilumination}

\author{
Kepa Balparda ${ }^{1,2 *}$, Ma. Isabel Maya ${ }^{3}$ y Carolina Mora ${ }^{4}$ \\ ${ }^{1}$ Ciencias de la Visión, Universidad de Valladolid, Valladolid, España; ${ }^{2}$ Córnea y Cirugía Refractiva, Black Mammoth Surgical, Medellín, Colombia; \\ ${ }^{3}$ Oftalmología, Universidad Pontificia Bolivariana, Medellín, Colombia; ${ }^{4}$ Medicina General, Universidad Pontificia Bolivariana, Medellín, Colombia
}

\section{Resumen}

Se presenta el caso de un paciente de género masculino de 31 años de edad, a quien se le diagnosticó distrofia polimorfa posterior, una entidad poco frecuente, secundaria a alteraciones a nivel de la cresta neural. Si bien la mayoría de los pacientes tiene afectación bilateral, no son raros los casos totalmente unilaterales, como el reportado en el presente artículo.

Palabras clave: Distrofia polimorfa posterior. Tomografía Scheimpflug. Membrana de Descemet. Endotelio corneal.

\begin{abstract}
We present the case of a 31-year-old male patient, who was diagnosed with posterior polymorphic dystrophy, a rare hereditary condition of the neural crest-derived. Although most patients have bilateral involvement, totally unilateral cases, such as the one reported in this article, are not rare.
\end{abstract}

Key words: Posterior polymorphous dystrophy. Scheimpflug tomography. Descemet's membrane. Corneal endothelium.

\section{Descripción del caso}

La distrofia polimorfa posterior (DPP) es una condición congénita rara, con herencia autosómica dominante'. Se deriva de alteraciones en el desarrollo a nivel de la cresta neural, dando origen a cambios anatómicos definidos que afectan principalmente la membrana de Descemet y el endotelio corneal ${ }^{2}$. Una de las morfologías más frecuentes de la DPP es el patrón «en banda» o «baba de caracol», la cual se presenta a continuación según su evaluación en un paciente asintomático de 31 años de edad, en quien se diagnosticó la distrofia de forma unilateral

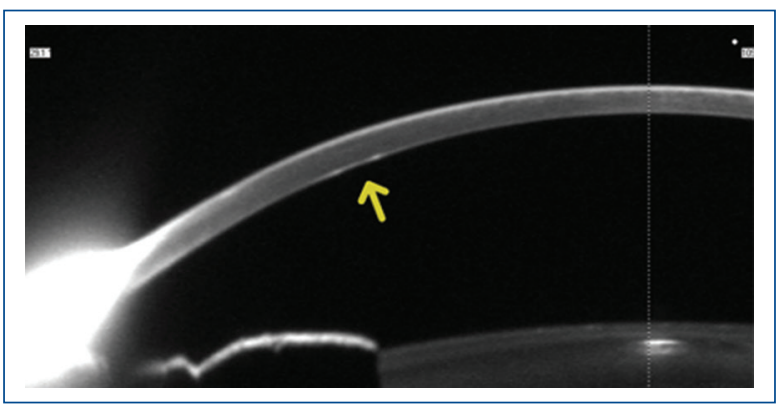

Figura 1. Imagen de tomografía Scheimpflug en ojo izquierdo que demuestra lesiones hiperdensas, en pareja, bien definidas, a nivel de la membrana de Descemet y el endotelio corneal (flecha). El resto de la imagen tomográfica es normal.

\section{Correspondencia:}

*Kepa Balparda

E-mail: kb@ kepabalparda.com
Fecha de recepción: 10-10-2021

Fecha de aceptación: 30-10-2021 DOI: 10.24875/RSCO.M21000017
Disponible en internet: 28-12-2021 Rev Soc Colomb Oftalmol. 2021;54(2):101-102 www. revistaSCO.com 0120-0453 / @ 2021 Sociedad Colombiana de Oftalmología (SOCOFTAL). Publicado por Permanyer. Este es un artículo open access bajo la licencia CC BY-NC-ND (http://creativecommons.org/licenses/by-nc-nd/4.0/). 


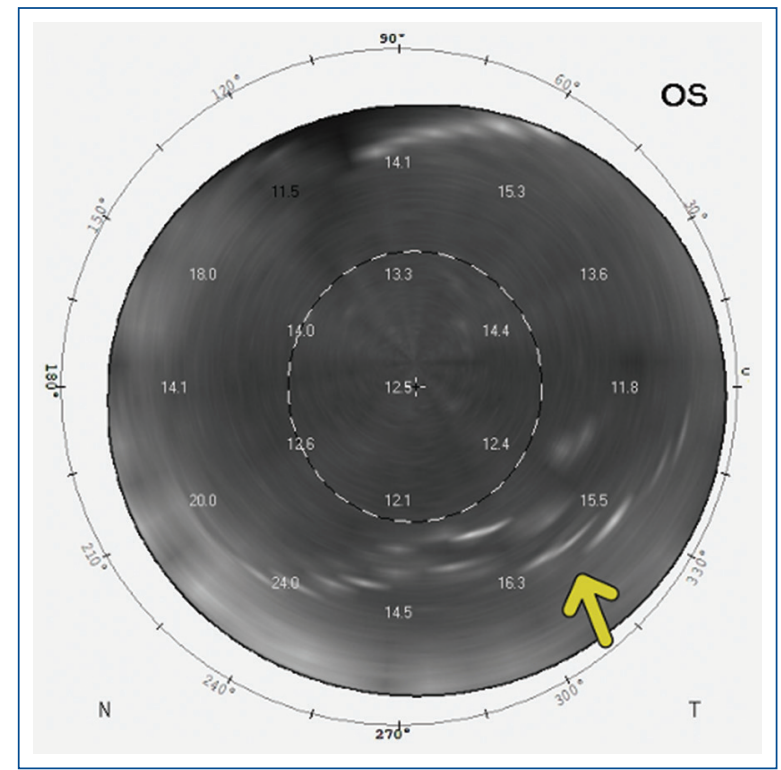

Figura 2. Imagen de densitometría corneal basada en tomografía Scheimpflug en ojo izquierdo, enfocada en las 60 micras más posteriores de la córnea. Se observan lesiones hiperdensas, en pareja, que afectan la media periferia inferior de la córnea (flecha).

en su ojo izquierdo como hallazgo incidental (Figs. 1-3).

\section{Financiamiento}

La presente investigación no ha recibido ninguna beca específica de agencias de los sectores publicos, comercial o con ánimo de lucro.

\section{Conflicto de intereses}

Los autores declaran no tener conflicto de intereses.

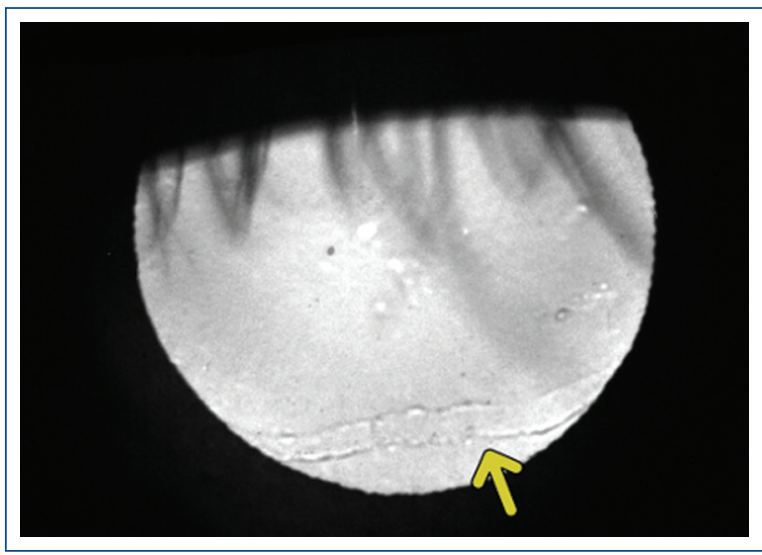

Figura 3. Imagen de retroiluminación ocular sin usar midriasis farmacológica. Se observan lesiones hiperdensas, en pareja, contorneadas, que afectan la media periferia inferior de la córnea (flecha).

\section{Responsabilidades éticas}

Protección de personas y animales. Los autores declaran que para esta investigación no se han realizado experimentos en seres humanos ni en animales.

Confidencialidad de los datos. Los autores declaran que han seguido los protocolos de su centro de trabajo sobre la publicación de datos de pacientes.

Derecho a la privacidad y consentimiento informado. Los autores han obtenido el consentimiento informado de los pacientes y/o sujetos referidos en el artículo. Este documento obra en poder del autor de correspondencia.

\section{Bibliografía}

1. Mendoza-Adam G, Hernández-Camarena JC, Valdez-García JE. Distrofia polimorfa posterior, presentación de un caso y análisis de la literatura. Arch Soc Esp Oftalmol. 2015;90(9):439-41.

2. Jang MS, Roldan AN, Frausto RF, Aldave AJ. Posterior polymorphous corneal dystrophy 3 is associated with agenesis and hypoplasia of the corpus callosum. Vision Res. 2014;100:88-92. 